\title{
The Beta-Adrenergic Receptors
}

\author{
JOHN P. PERKINS, ED. \\ Humana; 1991; Clifton, $\mathrm{NJ}$; \\ ISBN 0-89603-173-X; $\$ 89.50 ; 405$ pp.
}

Received July 19, 1991; Accepted July 19, 1991

Recently, tremendous strides have been made in characterization of $\beta$-adrenergic receptors including cloning and sequencing of these receptors, which are known to operate by regulating the activity of G-proteins. This book consists of chapters on identification and characterization, structure-function relationships, and mechanisms of ligand-induced desensitization of $\beta$-adrenergic receptors. These receptors have been purified to homogeneity by affinity chromatography and reconstituted into phospholipid vesicles together with G-proteins and adenylate cyclase.

In a chapter by Perkins, Handsdorff, and Lefkowitz, agonist-induced receptor phosphorylation involving kinases leading to rapid desensitization is described. Large-scale purification of both mammalian $\beta_{1}$ and $\beta_{2}$ adrenergic receptors as well as the isolation of the cDNAs and gene encoding of these receptors has been achieved. Malbon, Moxham, and Brandwein have reviewed the efforts to produce antibodies specific for $\beta$-adrenergic receptors and to apply immunochemical strategies to analyze these receptor molecules.

Autoradiographic studies of $\beta$-adrenergic receptors described by Wolfe reveal that $\beta$-adrenergic receptors and their subtypes are extremely heterogeneously distributed throughout most organs. Beta-adrenergic receptors in pathophysiological states and in clinical medicine have been described in a chapter by Insel. Stiles has highlighted several important facts concerning the pathophysiological regulation of the $\beta$-adrenergic receptor-adenylate cyclase system in a chapter on receptor regulation. Each of the components-the receptor, the G-proteins, and the catalytic unit-appears to be regulated by a variety of interventions.

Each chapter consists of an exhaustive review of pertinent literature as well as methodological details of the experiments discussed. The book 
would be useful for people working in the field of catecholamines in general and particularly for workers in the field of adrenergic receptor regulation and of signal transduction mechanisms.

Gopi A. Tejwani Department of Pharmacology College of Medicine Ohio State University Columbus, $\mathrm{OH} 43210-1239$ 


\title{
Advances in Neurology, vol. 53
}

\section{Parkinson's Disease: Anatomy, Pathology, and Therapy}

\author{
Max B. Streifler; Amos D. Korczyn, Eldad Melamed, \\ AND MOUSSA B.H. YOUDIM, EDS. \\ Raven; 1990; New York; \$125.00; 624 pp.
}

Received July 15, 1991; Accepted July 15, 1991

Our understanding of the etiology of Parkinson's disease, the developments of animal models, the studies of environmental factors, and revolutionary approaches to treatment represent but a few of the most exciting areas occurring in our understanding of Parkinson's disease. Undoubtedly among the degenerative diseases, for which the knowledge and understanding have skyrocketed in recent years, Parkinson's disease is the most likely example for the broadest approach utilizing many innovative techniques. This volume represents the compilation of papers presented at the 9th International Symposium on Parkinson's disease. The papers published in this volume range from thorough reviews of specific topics to reports on new experiments and clinical experiences. The chapters reflect the complete coverage of this diverse subject.

The volume begins with normal and pathological anatomy where the findings of light microscopy, electron microscopy, and immunochemistry are reviewed and the most recent observations are discussed. These chapters closely link the morphology with the chapters on neurochemistry and pharmacology. Chapters in this group are theoretically oriented as well as directly related to the rationale of various therapeutic pharmacological interventions. The neurophysiology section has basic research and clinical applications. The toxicology and environment section addresses, most importantly, the effect of MPTP in experimental animals, which represents a major breakthrough in techniques available for study of striatal pathology. The data indicating implications 
of importance of environmental factors in development of disease in humans are also addressed. The chapter on clinical aspects addresses the clinical expression of the disease both in its most common expressions as well as in the less common forms and provides most valuable data in illustrating the vagaries and nuances of clinical expressions. This is followed by a chapter that addresses the current therapy and its side effects. Since the introduction of L-dopa in the 1960s a considerable understanding of the responses to therapy has been gained, which is of course now augmented by the evaluation of the numerous new pharmaceutical approaches to the treatment of Parkinson's disease. Results of larger studies are of considerable clinical importance.

Finally, the wave of the future is extensively discussed. The chapter on therapeutic research and developments where both the reviews of our current status as well as the results of the early attempts of the adrenal transplants are discussed.

It is a well-organized volume, which despite the high number of contributors represents a considerable degree of uniformity. It definitely is an invaluable reference for medical practitioners dealing with Parkinson's disease which gives considerable guidance and allows for better understanding of the newer developments in the treatment of patients.

Despite the rapid progression of knowledge in all areas addressed by the presenters at the congress, these contributions are quite valid although some represent early reports on some newer research approaches. This book can be regarded as a must for neurologists, and is most valuable for all physicians treating Parkinson patients and for researchers who are addressing various facets of the pathogenesis, etiology, or treatment of this disease.

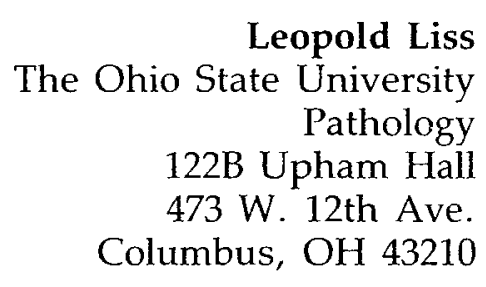

